\title{
Clinical significance of COL1A1 and COL1A2 expression levels in hypopharyngeal squamous cell carcinoma
}

\author{
PEILIANG LIN ${ }^{1,2^{*}}$, PENG TIAN ${ }^{1,2^{*}}$, JIAQI PANG ${ }^{1,2}$, LAN LAI $^{1,2}$, GUI HE $^{1,3}$, \\ YANG SONG $^{1,3}$ and YIQING ZHENG $^{1,2}$ \\ ${ }^{1}$ Guangdong Provincial Key Laboratory of Malignant Tumor Epigenetics and Gene Regulation; \\ ${ }^{2}$ Department of Otolaryngology; ${ }^{3}$ Cellular and Molecular Diagnostics Center, Sun Yat-sen Memorial Hospital, \\ Sun Yat-sen University, Guangzhou, Guangdong 510120, P.R. China
}

Received July 7, 2019; Accepted April 3, 2020

DOI: 10.3892/ol.2020.11594

\begin{abstract}
Alterations in collagen type I $\alpha 1$ (COL1A1) and collagen type I $\alpha 2$ (COL1A2) expression levels have been reported to predict prognosis in various types of cancer. However, the effect of these biomarkers on hypopharyngeal squamous cell carcinoma (HPSCC) is yet to be fully elucidated. The present study aimed to explore the prognostic significance of COL1A1 and COL1A2 expression levels in HPSCC. The expression levels of COL1A1 and COL1A2 in 67 patients with HPSCC were examined using an immunohistochemical assay in a tissue microarray. The associations between COL1A1/COL1A2 expression levels and patient clinicopathological features were analyzed using ANOVA, Pearson's $\chi^{2}$ or Fisher's exact test. The Cox proportional hazard models and Kaplan-Meier survival analysis with log-rank tests were used to analyze the significance of COL1A1/COL1A2 as prognostic markers for patients with HPSCC. As a result, immunohistochemical staining revealed that COL1A1 was positively expressed in all cases, among which $40.3 \%$ were strong positive, while COL1A2 was positively expressed in $76.1 \%$ of the HPSCC cases with $6.0 \%$ of the samples exhibiting strong staining. Further analysis revealed no significant association between the expression levels of COL1A1/COL1A2 and other clinicopathological features. Cox regression analysis revealed that a high COL1A2 expression level predicted a high locoregional recurrence and a less favorable disease-free survival rate $(\mathrm{P}=0.042$ and 0.020 , respectively). Overall, the present study indicated that COL1A2 expression levels may have value as a prognostic indicator in HPSCC.
\end{abstract}

Correspondence to: Professor Yiqing Zheng, Guangdong Provincial Key Laboratory of Malignant Tumor Epigenetics and Gene Regulation, Sun Yat-sen Memorial Hospital, Sun Yat-sen University, 107 Yanjiang West Road, Guangzhou, Guangdong 510120, P.R. China E-mail: zhengyiq@mail.sysu.edu.cn

*Contributed equally

Key words: hypopharyngeal squamous cell carcinoma, COL1A1, COL1A2, tissue microarray, prognosis

\section{Introduction}

Hypopharyngeal squamous cell carcinoma (HPSCC) is associated with one of the worst prognoses of all types of upper aerodigestive tract cancer based on the U.S. population-based analysis $(1,2)$. Due to a rich network of lymphatic vessels and the late presentation of symptoms, HPSCC is usually detected at an advanced stage (3). Surgery and radiotherapy alone, or in conjunction with chemoradiation, is the standard treatment for HPSCC. However, the overall 5-year survival rate for hypopharyngeal cancer is unsatisfactory $(37.5-41.3 \%)(2)$. The identification of novel prognostic biomarkers may optimize the risk stratification systems of HPSCC and, thus, may improve clinical decision-making to ultimately improve patient survival.

Type I collagen is an important member of the collagen family, which is a key structural component of the extracellular matrix (4). Type I collagen typically consists of a heterotrimer of two chains of collagen type I $\alpha 1$ (COL1A1) and one chain of collagen type I $\alpha 2$ (COL1A2) (5). Type I collagen is considered to influence tumor invasion and progression $(6,7)$. Recently, abnormal expression levels of COL1A1 and COL1A2 have been reported in several types of cancer (8-10). COL1A2 is downregulated in melanoma and bladder cancer, while COL1A1 and COL1A2 mRNA expression levels are upregulated in colorectal cancer and medulloblastoma (5,10-12). In addition, Misawa et al (13) reported that the methylation of the COL1A2 gene was an independent adverse prognostic factor in head and neck cancer (13); however, this group have not investigated the prognostic significance of COL1A2 in hypopharyngeal squamous cell carcinoma, in detail.

In the present study, immunohistochemistry (IHC) was used to evaluate the protein expression levels of COL1A1 and COL1A2 in hypopharyngeal squamous cell carcinoma tissues and to explore the prognostic significance of these proteins in HPSCC.

\section{Materials and methods}

Patients and specimens. Between April 2012 and March 2017, 67 patients, who were newly diagnosed with primary HPSCC and underwent radical surgery at the Sun Yat-sen Memorial Hospital (Guangzhou, China), were enrolled in the present 
study. The mean age of the patients was 58.3 years (range of 41.0-74.0 years) with 97 and 3\% males and females, respectively. The cases selected were based on the following criteria: i) No previous malignant disease or a second primary tumor; and ii) no previous history of treatment with radiotherapy, chemotherapy or surgery before HPSCC was diagnosed. The pathological Tumor-Node-Metastasis (TNM) stage was defined based on the 2017 edition of the TNM classification of the American Joint Committee on Cancer (14). All the experiments carried out in this study were approved by The Ethics Committee of Sun Yat-sen Memorial Hospital, Sun Yat-sen University (Guangzhou, China).

Tissue samples were obtained during the radical surgery and were dehydrated at $4^{\circ} \mathrm{C}(70,80,90$ and $95 \%$ ethanol, $3 \mathrm{~h}$ for each step, and followed by $100 \%$ ethanol twice, $2 \mathrm{~h}$ for each step), and vitrified (100\% xylene twice, $0.5 \mathrm{~h}$ for each step). Subsequently, tissue samples were immersed in melting paraffin at $56-58^{\circ} \mathrm{C}$ for $0.5 \mathrm{~h}$ and three times and then embedded in paraffin.

Tissue microarray construction. The paraffin-embedded tissue blocks were cut into 4- $\mu \mathrm{m}$ thick sequential sections and the slides were dried, deparaffinized in xylene at $20^{\circ} \mathrm{C}$, rehydrated using a graded ethanol series (100, 95, 80 and 70\% ethanol followed by $\mathrm{ddH}_{2} \mathrm{O}, 2 \mathrm{~min}$ for each step) and immersed in hematoxylin (Sigma-Aldrich; Merck KGaA) at $20^{\circ} \mathrm{C}$ for 10 min followed by $\mathrm{ddH}_{2} \mathrm{O}$ for $2 \mathrm{~min}$. Subsequently, the slides were differentiated with acidic ethanol for $15 \mathrm{sec}$ and washed in running water for $30 \mathrm{~min}$. The slides were sequentially immersed in eosin at $20^{\circ} \mathrm{C}$ for $2 \mathrm{~min}$ followed by $\mathrm{ddH}_{2} \mathrm{O}$ for $2 \mathrm{~min}$. Finally, the sections were dehydrated (70, 80, 90 and 100\% ethanol, 2 min for each step), vitrified (100\% ethanol, followed by $50 \%$ ethanol and $50 \%$ xylene, then $100 \%$ xylene) and mounted with resinene (Biosharp Life Sciences). Hematoxylin and eosin-stained slides were reviewed and the tumor zone in the paraffin-embedded specimens was selected for tissue microarray (TMA) construction. Tissue microarrays were constructed in accordance with a previously described method (15). For each case, two cores taken from the selected tumor area were used to construct the TMA. A hollow needle was used to punch and remove bipartite cylinders tissue cores (1.0 mm diameter) from selected donor tissue regions. Further, the punched tissue cores were inserted into a recipient paraffin block with a precisely spaced array pattern, using a manual tissue arraying instrument according to the manufacturer's protocol (Beecher Instruments, Silver Spring, Maryland, USA).

Immunohistochemistry (IHC). IHC was performed using the standard EnVision method (16). The paraffin-embedded tissue blocks were cut into $4-\mu \mathrm{m}$ thick sequential sections and the slides were dried, deparaffinized in xylene at $20^{\circ} \mathrm{C}$, rehydrated using a graded ethanol series $(100,95,80$ and $70 \%$ ethanol and followed by $\mathrm{dd}_{2} \mathrm{O}$ ) and immersed in $3 \%$ hydrogen peroxide for $10 \mathrm{~min}$ at $20^{\circ} \mathrm{C}$ to block endogenous peroxidase activity. Antigens were retrieved by pressure cooking at $120^{\circ} \mathrm{C}$ for $3 \mathrm{~min}$ in citrate buffer ( $\mathrm{pH}$ 6). The slides were then incubated with $5 \% \mathrm{BSA}$ at $20^{\circ} \mathrm{C}$ for $15 \mathrm{~min}$ to prevent the non-specific reaction. Subsequently, the slides were incubated with a primary antibody at $4^{\circ} \mathrm{C}$ overnight. The slides were sequentially incubated with a secondary antibody for $30 \mathrm{~min}$ in an incubator at $37^{\circ} \mathrm{C}$ and stained with 3,3'-diaminobenzidine.
Finally, the sections were counterstained with Mayer's hematoxylin (Sigma-Aldrich; Merck KGaA), dehydrated (70, 80, 90 and $100 \%$ ethanol, 2 min for each step), vitrified (100\% ethanol, followed by $50 \%$ ethanol and $50 \%$ xylene, then $100 \%$ xylene) and mounted with resinene (Biosharp Life Sciences). A negative control was obtained by replacing the primary antibody with a normal rabbit immunoglobulin $\mathrm{G}$.

The primary antibodies used in the present study were polyclonal rabbit anti-human COL1A2 (cat.no. YT1019; 1:200) and monoclonal mouse anti-human COL1A1 (1:200; cat. no. YM3767) (both ImmunoWay Biotechnology Company).

IHC evaluation. The positively stained cells were brown or yellow in color. Immune reactivity was scored by evaluating the number of positive cells and the positive intensity score using light microscopy at x100 magnification. The percentage of positive tumor cells corresponded to the following scores: i) 0, staining in $<1 \%$ of tumor cells; ii) 1 , staining in $1-25 \%$; iii) 2 , staining in $26-50 \%$; iv) 3 , staining in $51-75 \%$; and v) 4 , staining in $>75 \%$ of tumor cells. The positive intensity score was defined as follows: i) 0 , no expression; ii) 1 , weak; iii) 2 , mild; iv) 3 , moderate; and v) 4 , strong. The total score, ranging from $0-16$, was obtained by multiplying the proportion and intensity scores. The IHC results were defined as negative with a total score of 0 , mild positive with a total score of 1-8 and strong positive with a total score of 9-16. Two researchers who were blinded to the information of the patients performed the scoring. If the results differed, a third researcher would then participate to confirm the score.

Statistical analysis. Qualitative variables were summarized as absolute and relative frequency (percentage). Quantitative variables were summarized as means and standard deviations (mean \pm standard deviation). SPSS software (version 21.0; IBM Corp) was used to perform the analysis. Pearson's $\chi^{2}$ or the Fisher's exact test were applied to evaluate the association between COL1A1/COL1A2 expression levels and the categorical clinicopathological variables of patients with HPSCC (data not shown). One-way ANOVA was applied to evaluate the association between COL1A1/COL1A2 expression levels with the quantitative variables of the clinicopathological features of patients with HPSCC. If significant association was found, Scheffe post hoc test would be performed (data not shown). The follow-up time was defined as the time period between the day of surgery and the end of follow-up or death. The follow up was conducted in outpatient department. The frequency of follow up was once every 3 months during the first two years, once every 6 months during the third to fifth year, and once every year after 5 years. The follow-up period ranged from 5-61 months, with a median follow-up period of 18 months. Cox proportional hazard models were used to investigate the associations between tumor recurrence/survival rate and certain risk factors. Kaplan-Meier survival analysis was also performed with log-rank tests based on different COL1A2 staining. $\mathrm{P}<0.05$ was considered to indicate a statistically significant difference.

\section{Results}

Patient characteristics. The characteristics and pathological features of the patients are presented in Table I. Overall, 46/67 
Table I. Clinicopathological characteristics of patients with hypopharyngeal squamous cell carcinoma $(n=67)$.

\begin{tabular}{lc}
\hline Parameter & Value \\
\hline Age, years, mean \pm standard deviation & $58.3 \pm 8.0$ \\
Sex, n $(\%)$ & \\
Male & $65(97.0)$ \\
Female & $2(3.0)$ \\
Hypertension, n $(\%)$ & \\
Yes & $10(14.9)$ \\
No & $57(85.1)$
\end{tabular}

Diabetes Mellitus, n (\%)

Yes

$1(1.4)$

No

$66(98.6)$

Smoker, n (\%)

Yes

$46(68.7)$

No

$21(31.3)$

Alcohol consumption, n (\%)

Yes

$34(50.7)$

No

$33(49.3)$

T classification, $\mathrm{n}(\%)$

T1

T2

T3

$\mathrm{T} 4 \mathrm{a}$

$\mathrm{T} 4 \mathrm{~b}$

$8(11.9)$

$15(22.4)$

$24(35.8)$

$17(25.4)$

$3(4.5)$

Histological grade, n (\%)

G1

$24(35.8)$

G2

G3

G4

$29(43.3)$

$13(19.4)$

1 (1.5)

Lymphovascular invasion, n (\%)

Yes

$11(16.4)$

No

$56(83.6)$

Margin of removed primary lesion, $\mathrm{n}(\%)$

Clear margin

$54(80.6)$

Close margin

$11(16.4)$

$2(3.0)$

Involved margin

$\mathrm{N}$ classification, $\mathrm{n}(\%)$

N0

$16(23.9)$

$\mathrm{N} 1$

$12(17.9)$

1 (1.5)

$19(28.4)$

18 (26.8)

$1(1.5)$

N3

$3(4.5)$

$39(58.2)$

$20(29.9)$

Iva

$1(1.5)$

Ivb
Table I. Continued.

\begin{tabular}{lc}
\hline Parameter & Value \\
\hline Treatment, n (\%) & \\
Sx+CRT & $34(50.7)$ \\
Sx+RT & $9(13.4)$ \\
Sx+CT & $5(7.5)$ \\
Sx & $19(28.4)$
\end{tabular}

T, Tumor; N, node; M, metastasis; Sx, surgery; CRT, chemoradiotherapy; RT, radiotherapy; CT, chemotherapy.

(68.7\%) of the patients were smokers and 34/67 (50.7\%) were positive for alcohol consumption. The primary tumors of 44/67 (65.7\%) of the patients were staged as T3-4 according to the final pathological results. Histological grades in 53/67 (79.1\%) of patients were grade 1 or 2 . Excisional margins in $80.6 \%$ of the patients were clear margins, whereas those in $3.0 \%$ patients were involved margins. The regional lymph node stage of 38 $(56.7 \%)$ of the patients was N2. The anatomic stage of $89.6 \%$ the patients was Stage III-IV. Overall, $71.6 \%$ of patients had undergone adjuvant radiotherapy or/and chemotherapy. The 5 -year locoregional recurrence and distant metastasis rate were 38 and $50 \%$, respectively. The 5-year overall survival rate was $37 \%$, while the 5 -year disease-free survival rate was $22 \%$.

COL1A1 and COL1A2 expression patterns in HPSCC tissues. Both COL1A1 and COL1A2 expression levels were evaluated in the tumor parenchyma component, which were primarily a cytoplasmic pattern. COL1A1 expression was mildly positive in $40(59.7 \%)$ of the tumors, while $27(40.3 \%)$ of the tumors had a strong COL1A1 expression. COL1A2 did not express in $16(23.9 \%)$ of the tumors, while $47(70.1 \%)$ of the tumors had a mild COL1A2 expression and $4(6.0 \%)$ had a strong COL1A2 expression. Representative immunohistochemical images of COL1A1 and COL1A2 expression in HPSCC tissues are presented in Figs. 1 and 2.

Association of COL1A1/COL1A2 expression levels with the clinicopathological features and survival of the patients with HPSCC. Pearson's $\chi^{2}$, Fisher's exact test and one-way ANOVA test revealed no significant associations between COL1A1/COL1A2 expression levels and patient clinicopathological features (data not shown). Univariate cox regression analysis revealed that the expression level of COL1A2 was a significant prognostic factor for locoregional recurrence and disease-free survival rate ( $\mathrm{P}=0.042$ and 0.020 , respectively). $\mathrm{N}$ status was not significantly associated with disease-free survival rate or overall survival rate $(\mathrm{P}=0.094$ and 0.075 , respectively). COL1A2 expression levels were not significantly associated with overall survival rate $(\mathrm{P}=0.052)$. No other clinicopathological features significantly predicted locoregional recurrence, distant metastasis, disease-free survival rate or overall survival rate (Table II).

The Kaplan-Meier survival curves revealed that the curves were separated between the negative and positive COL1A2 staining curves for locoregional control rate and disease-free survival rate, although Log-rank tests revealed no statistical 


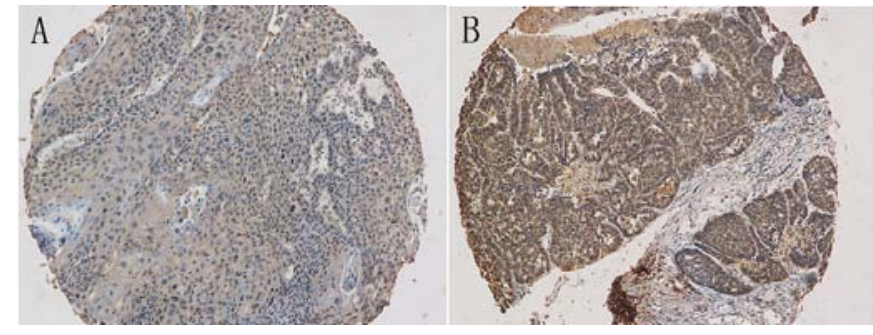

Figure 1. Representative immunohistochemical images with collagen type I $\alpha 1$ expression levels in hypopharyngeal squamous cell carcinoma Magnification, x100. (A) Mild staining. (B) Strong staining.

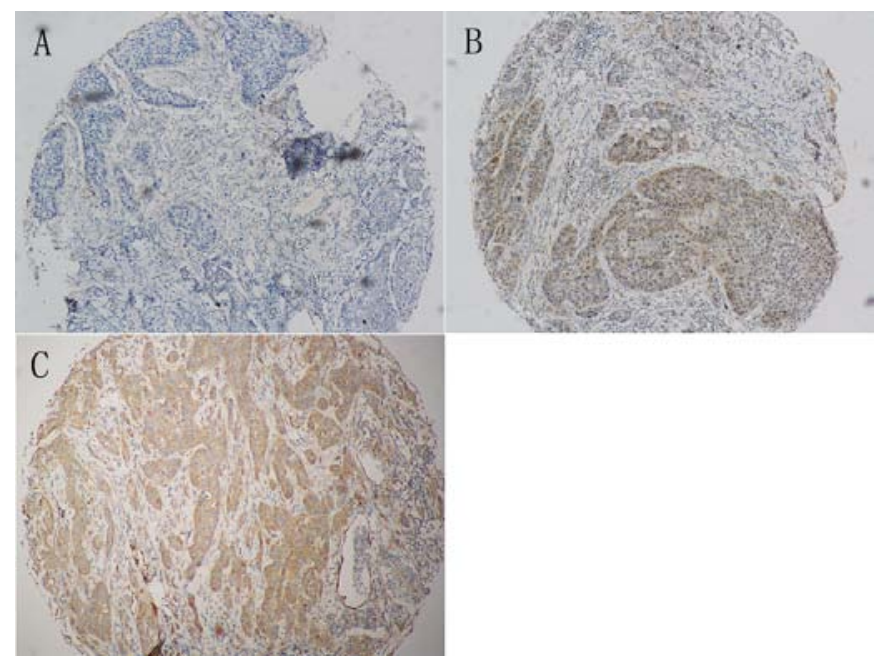

Figure 2. Representative immunohistochemical images with collagen type I $\alpha 2$ expression in hypopharyngeal squamous cell carcinoma. Magnification, x100. (A) Negative staining. (B) Mild staining. (C) Strong staining.

significance (Fig. 3A-D). As the COL1A2 staining results were calculated as a binary variable in Kaplan-Meier survival analysis using log-rank tests, the test was not as reliable as the Cox regression analysis, in which the COL1A2 staining results were calculated as a continuous variable.

\section{Discussion}

Although the hypopharynx is one of the least common subsites for head and neck cancer, HPSCC has a poor prognosis (5-yeaer survival rate, $37.5-41.3 \%)(2,17)$. Since the hypopharynx is rich in highly anastomotic regional lymphatics, HPSCC disseminates into the nodal basins of the neck quickly (18). Thus, these tumors often present at an advanced stage. In published studies, the 5-year survival rates of patients with HPSCC have been reported to range from $24-33 \%$ post-surgery alone and from $35-52 \%$ for post-combined surgery and radiation therapy $(19,20)$. Advances in treatment techniques may improve the survival rate; however, risk stratification is also important in developing an individualized treatment management schedule. To date, researchers have made efforts to identify prognostic factors, including clinical features (21), cellular adverse factors $(22,23)$ and molecular markers $(24,25)$. This information can be used to identify patients with high-risk HNSCC who may benefit from more aggressive therapy and frequent follow-up following primary treatment.
Recently, the role of the microenvironment in tumorigenesis and tumor progression has been a focus of research. It is hypothesized that epithelial cell functions, including cellular differentiation, migration and invasion, are regulated via interaction with the extracellular matrix (ECM) (26). Type I collagen is a major structural component of the ECM. It has been reported that type I collagen remodeling in the ECM microenvironment accompanies stromal invasion and epithelial tumorigenesis in various types of cancer (27). Type I collagen is composed of an $\alpha 1$ chain and $\alpha 2$ chain. Abnormal expression of the $\alpha 1$ chain and/or $\alpha 2$ chain impairs the function of type I collagen and tissue homeostasis (5). The role of COL1A2 in various types of cancer remains controversial. On the one hand, Bonazzi et al (28) and Mori et al (5) reported that COL1A2 was downregulated in melanoma and bladder cancer. On the other hand, COL1A2 was observed to be upregulated in colorectal cancer (11), gastric cancer $(29,30)$, breast cancer (31) and medulloblastoma (12). Koga et al (4) reported that COL1A2 methylation was predominantly detected in advanced stage melanoma tumors. Mori et al (5) reported that $\mathrm{CpG}$ hypermethylation inactivated the COL1A2 gene and promoted the proliferative and migratory activity of bladder cancer. In a study by Misawa et al (13), hypermethylation of the COL1A2 promoter occurred with a high frequency and the expression levels of COL1A2 was downregulated in HNSCC cell lines. In addition, disease-free survival rate was significantly less favorable in patients with methylation in COL1A2. However, in the present study, the upregulated expression of COL1A2 was revealed to be associated with a high locoregional recurrence and a less favorable disease-free survival rate in HPSCC. One of the main differences between the two studies was that the present study primarily focused on patients with HPSCC, while Misawa et al (13) grouped HPSCC with oropharyngeal, laryngeal and other types of cancer, and HPSCC compiled $25 \%$ of cases. This may have resulted in the different conclusion of the two studies.

There were certain limitations to the present study. As a preliminary study, the present study only assessed the expression levels of COL1A1 and COL1A 2 using IHC in HPSCC and analyzed the association between the expression levels of COL1A1/COL1A2 and the clinicopathological characteristics, relapse and survival rate of the patients. Yet, previous studies have demonstrated a reciprocal interaction between urothelial carcinoma cells and the stromal compartment, which modulates the epithelial differentiation and progression of bladder cancer via paracrine signaling pathways, such as the Sonic hedgehog and Wnt/bone morphogenic pathways $(6,7)$. The mechanisms via which COL1A2 expression influences the locoregional recurrence and disease-free survival rate in HPSCC, and whether it reacts with other biomarkers, remains unclear; thus, further studies are required to elucidate these mechanisms. In the near future, in vivo and in vitro experiments should be performed to investigate whether regulation of COL1A2 expression could influence expression of other proteins and affect cancer cell migration, invasion and tumor progression. Due to the relative rarity of hypopharyngeal types of cancer, the patients included in the present study were relatively small in number and certain results should be confirmed in further studies with a larger population size. 


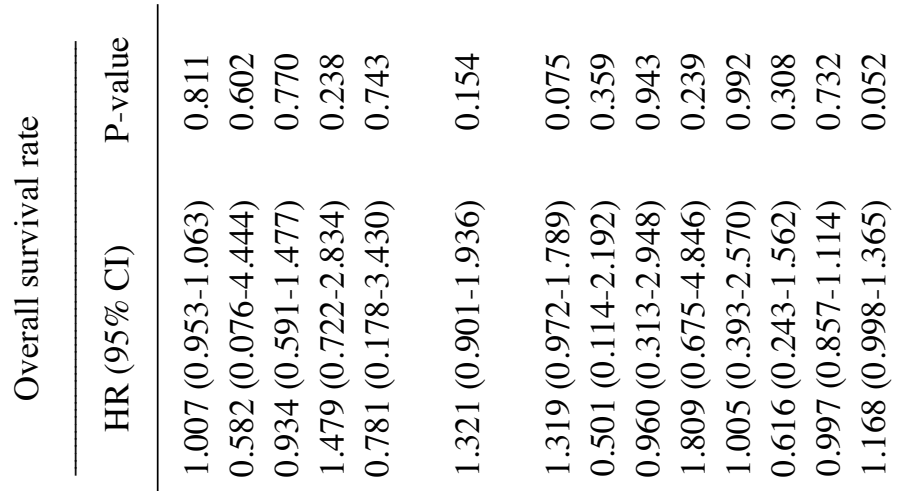

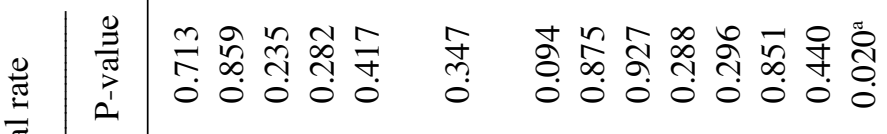

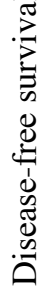

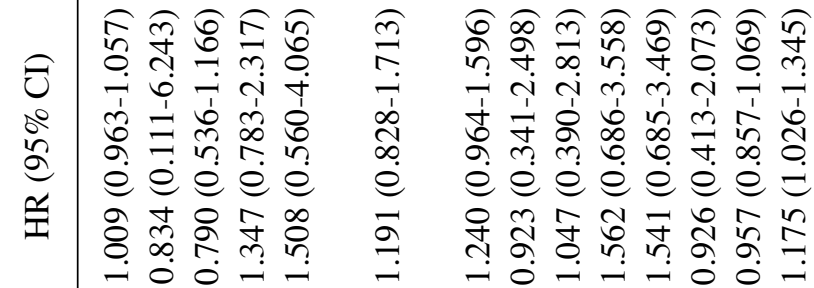

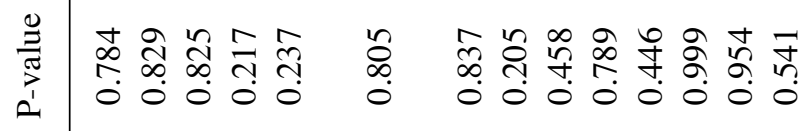

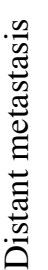

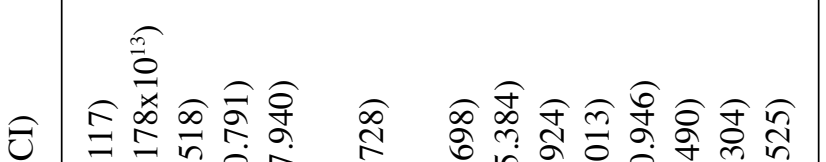

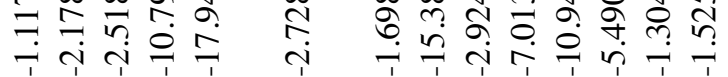

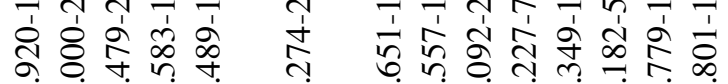

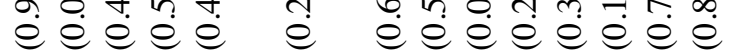

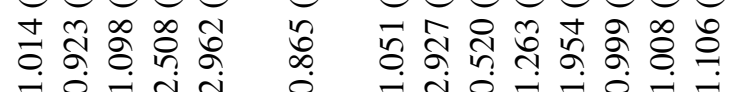

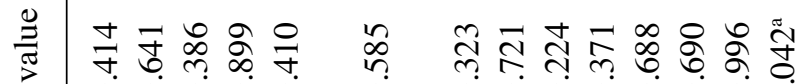
00000 - 00000000

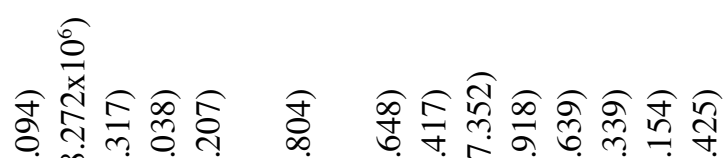
$\rightarrow \infty-i$ त

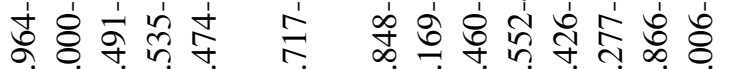
$\dot{e} \dot{e} e \dot{e} e \dot{e} e \hat{e} \dot{e}$ तิธ兀 엄 

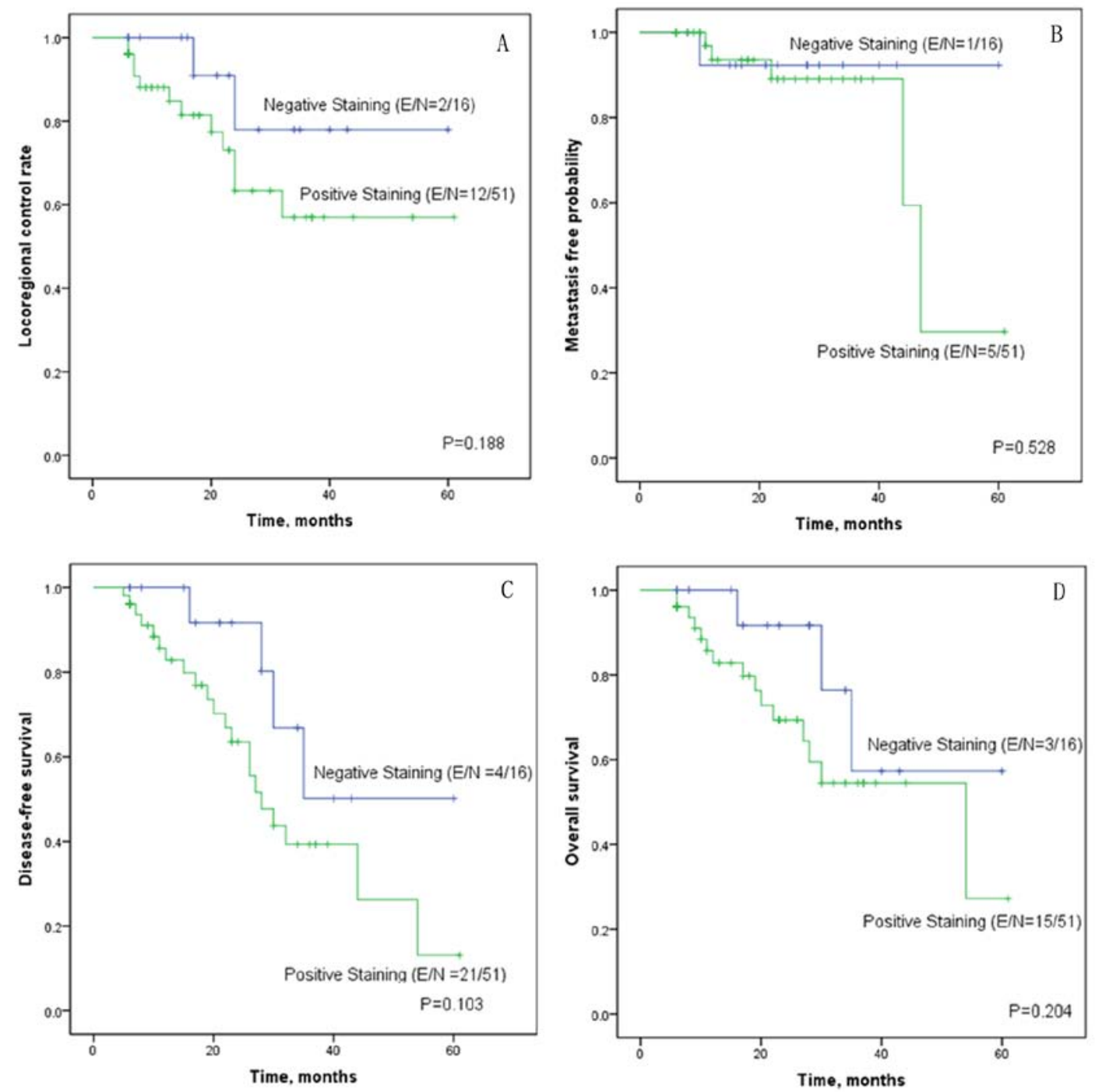

Figure 3. Kaplan-Meier survival plots for patients with hypopharyngeal squamous cell carcinoma. Survival plots show (A) locoregional control rate, (B) distant metastasis-free probability, (C) disease-free survival rate and (D) overall survival rate according to collagen type I $\alpha 2$ staining results. E/N indicates the number of events/total number of patients. The number of events refers to the number of the cases having locoregional relapse (A), distant metastasis (B), locoregional relapse/distant metastasis/death (C), and death (D), respectively.

In conclusion, the expression levels of COL1A2 have value as a prognostic factor of HPSCC in risk stratification and may assist in individual clinical management for patients with HPSCC in the future.

\section{Acknowledgements}

Not applicable.

\section{Funding}

The present study was supported by The National Natural Science Foundation of China (grant nos. 81570935 and 81771018).

\section{Availability of data and materials}

The datasets used and/or analyzed during the current study are available from the corresponding author on reasonable request.

\section{Authors' contributions}

PL and PT designed the study, analyzed the data and wrote the manuscript. JP and LL acquired the general data of the patients and assisted in the analysis of data. GH and YS performed tissue microarray construction, immunohistochemical staining and scoring. YZ was involved in designing the study, analyzing the data and reviewing the manuscript. All authors read and approved the final manuscript.

\section{Ethics approval and consent to participate}

The present study was approved by The Ethics Committee of Sun Yat-sen Memorial Hospital, Sun Yat-sen University (approval no. SYSEC-KY-KS-2018-155). Patients who participated in this research had complete clinical data. Written informed consent was provided by the patients or their guardians. 


\section{Patient consent for publication}

Not applicable.

\section{Competing interests}

The authors declare that they have no competing interests.

\section{References}

1. Pingree TF, Kim DR, Owen R and Larry D: Treatment of hypopharyngeal carcinoma: A 10-year review of 1,362 cases. Laryngoscope 97: 901-904, 1987.

2. Newman JR, Connolly TM, Illing EA, Kilgore ML, Locher JL and Carroll WR: Survival trends in hypopharyngeal cancer: A population-based review. Laryngoscope 125: 624-629, 2015.

3. Chu PY and Chang SY: Reconstruction of the hypopharynx after surgical treatment of squamous cell carcinoma. J Chin Med Assoc 72: 351-355, 2009.

4. Koga Y, Pelizzola M, Cheng E, Krauthammer M, Sznol M, Ariyan S, Narayan D, Molinaro AM, Halaban R and Weissman SM: Genome-wide screen of promoter methylation identifies novel markers in melanoma. Genome Res 19: 1462-1470, 2009

5. Mori K, Enokida H, Kagara I, Kawakami K, Chiyomaru T, Tatarano S, Kawahara K, Nishiyama K, Seki N and Nakagawa M: CpG hypermethylation of collagen type I alpha 2 contributes to proliferation and migration activity of human bladder cancer. Int J Oncol 34: 1593-1602, 2009.

6. Shin K, Lim A, Zhao C, Sahoo D, Pan Y, Spiekerkoetter E, Liao JC and Beachy PA: Hedgehog signaling restrains bladder cancer progression by eliciting stromal production of urothelial differentiation factors. Cancer Cell 26: 521-533, 2014.

7. Shin K, Lee J, Guo N, Kim J, Lim A, Qu L, Mysorekar IU and Beachy PA: Hedgehog/Wnt feedback supports regenerative proliferation of epithelial stem cells in bladder. Nature 472: 110-114, 2011.

8. Gao F, Li M, Xiang R, Zhou X, Zhu L and Zhai Y: Expression of CLDN6 in tissues of gastric cancer patients: Association with clinical pathology and prognosis. Oncol Lett 17: 4621-4625, 2019.

9. Zhai JM, An YH, Wang W, Fan YG and Yao GL: IL-32 expression indicates unfavorable prognosis in patients with colon cancer. Oncol Lett 17: 4655-4660, 2019.

10. Zhu YZ, Zhou K, Ruan LL, Sun F, Wang G and Li WF: Metadherin overexpression in perihilar cholangiocarcinoma is associated with lymph node metastasis and poor prognosis. Oncol Lett 17: 4514-4520, 2019.

11. Zou X, Feng B, Dong T, Yan G, Tan B, Shen H, Huang A, Zhang X, Zhang M, Yang P, et al: Up-regulation of type I collagen during tumorigenesis of colorectal cancer revealed by quantitative proteomic analysis. J Proteomics 94: 473-485, 2013.

12. Liang Y, Diehn M, Bollen AW, Israel MA and Gupta N: Type I collagen is overexpressed in medulloblastoma as a component of tumor microenvironment. J Neurooncol 86: 133-141, 2008.

13. Misawa K, Kanazawa T, Misawa Y, Imai A, Endo S, Hakamada K and Mineta $\mathrm{H}$ : Hypermethylation of collagen $\alpha 2$ (I) gene (COL1A2) is an independent predictor of survival in head and neck cancer. Cancer Biomark 10: 135-144, 2011.

14. National Comprehensive Cancer Network. (NCCN) clinical practice guidelines in oncology. Head and Neck Cancer, Version 1, 2019.

15. Kononen J, Bubendorf L, Kallioniemi A, Bärlund M Schraml P, Leighton S, Torhorst J, Mihatsch MJ, Sauter G and Kallioniemi OP: Tissue microarrays for high-throughput molecular profiling of tumor specimens. Nat Med 4: 844-847, 1998.

16. Chen J, Li S, Xiao Y, Zou X, Zhang X, Zhu M, Cai M and Xie D: p53R2 as a novel prognostic biomarker in nasopharyngeal carcinoma. BMC Cancer 17: 846, 2017.
17. Carvalho AL, Nishimoto IN, Califano JA and Kowalski LP: Trends in incidence and prognosis for head and neck cancer in the United States: A site-specific analysis of the SEER database. Int J Cancer 114: 806-816, 2005.

18. Koo BS, Lim YC, Lee JS, Kim YH, Kim SH and Choi EC: Management of contralateral N0 neck in pyriform sinus carcinoma. Laryngoscope 116: 1268-1272, 2006.

19. Kuo P, Chen MM, Decker RH, Yarbrough WG and Judson BL: Hypopharyngeal cancer incidence, treatment, and survival: Temporal trends in the United States. Laryngoscope 124: 2064-2069, 2014.

20. Wang YL, Feng SH, Zhu J, Zhu J, Zhu GP, Li DS, Wang Y, Zhu YX, Sun GH and Ji QH: Impact of lymph node ratio on the survival of patients with hypopharyngeal squamous cell carcinoma: A population-based analysis. PLoS One 8: e56613, 2013.

21. Abrahão R, Anantharaman D, Gaborieau V, Abedi-Ardekani B, Lagiou P, Lagiou A, Ahrens W, Holcatova I, Betka J, Merletti F, et al: The influence of smoking, age and stage at diagnosis on the survival after larynx, hypopharynx and oral cavity cancers in Europe: The ARCAGE study. Int J Cancer 143: 32-44, 2018.

22. Yang J, Hsueh CY, Cao W and Zhou L: Pretreatment lymphocyte-to-monocyte ratio as an independent prognostic factor for hypopharyngeal squamous cell carcinoma. Acta Otolaryngol 138: 734-740, 2018.

23. Kuwahara T, Takahashi H, Sano D, Matsuoka M, Hyakusoku H, Hatano T, Hiiragi Y and Oridate N: Fibrinogen and neutrophil-to-lymphocyte ratio predicts survival in patients with advanced hypopharyngeal squamous cell carcinoma. Anticancer Res 38: 5321-5330, 2018

24. Chen Y, Liu Y, Ni H, Ding C, Zhang X and Zhang Z: FoxM1 overexpression promotes cell proliferation and migration and inhibits apoptosis in hypopharyngeal squamous cell carcinoma resulting in poor clinical prognosis. Int J Oncol 51: 1045-1054, 2017.

25. Wu H, Chen J, Wang Q, Yin Y, Da P, Le H, Zhang Z and Qiu X: Abnormal expression of HAX-1 is associated with cellular proliferation and migration in human hypopharyngeal squamous cell carcinoma. Mol Med Rep 16: 4664-4670, 2017.

26. Lu P, Weaver VM and Werb Z: The extracellular matrix: A dynamic niche in cancer progression. J Cell Biol 196: 395-406, 2012.

27. Egeblad M, Rasch MG and Weaver VM: Dynamic interplay between the collagen scaffold and tumor evolution. Curr Opin Cell Biol 22: 697-706, 2010.

28. Bonazzi VF, Nancarrow DJ, Stark MS, Moser RJ, Boyle GM, Aoude LG, Schmidt C and Hayward NK: Cross-platform array screening identifies COL1A2, THBS1, TNFRSF10D and UCHL1 as genes frequently silenced by methylation in melanoma. PLoS One 6: e26121, 2011.

29. Zhuo C, Li X, Zhuang H, Tian S, Cui H, Jiang R, Liu C, Tao R and Lin X: Elevated THBS2, COL1A2, and SPP1 expression levels as predictors of gastric cancer prognosis. Cell Physiol Biochem 40: 1316-1324, 2016

30. Rong L, Huang W, Tian S, Chi X, Zhao P and Liu F: COL1A2 is a novel biomarker to improve clinical prediction in human gastric cancer: Integrating bioinformatics and meta-analysis. Pathol Oncol Res 24: 129-134, 2018

31. Lin J, Goldstein L, Nesbit A and Chen MY: Influence of hormone receptor status on spinal metastatic lesions in patients with breast cancer. World Neurosurg 85: 42-48, 2016.

This work is licensed under a Creative Commons Attribution-NonCommercial-NoDerivatives 4.0 International (CC BY-NC-ND 4.0) License. 